\title{
OsteoporosAtlas: a human osteoporosis-related gene database
}

\author{
Xun Wang Equal first author, 1 , Lihong Diao ${ }^{\text {Equal first author, } 1 \text {, Dezhi Sun }}{ }^{1}$, Dan Wang ${ }^{1}$, Jiarun Zhu ${ }^{1}$, Yangzhige He ${ }^{1}$, Yuan Liu ${ }^{1}$, \\ Hao Xu ${ }^{1}$, Yi Zhang ${ }^{1}$, Jinying Liu ${ }^{2}$, Yan Wang ${ }^{1}$, Fuchu He ${ }^{1}$, Yang Li ${ }^{\text {Corresp., } 1}$, Dong Li ${ }^{\text {Corresp. } 1}$ \\ ${ }^{1}$ Beijing Proteome Research Center, Beijing Institute of Life omics, Beijing, China \\ 2 School of Traditional Chinese Medicine., Beijing University of Chinese Medicine, Beijing, China \\ Corresponding Authors: Yang Li, Dong Li \\ Email address: liyang_bprc@163.com, lidong.bprc@foxmail.com
}

Background. Osteoporosis is a common, complex disease of bone with a strong heritable component, characterized by low bone mineral density, microarchitectural deterioration of bone tissue and an increased risk of fracture. Due to limited drug selection for osteoporosis and increasing morbidity, mortality of osteoporotic fractures, osteoporosis has become a major health burden in aging societies. Current researches for identifying specific loci or genes involved in osteoporosis contribute to a greater understanding of the pathogenesis of osteoporosis and the development of better diagnosis, prevention and treatment strategies. However, little is known about how most causal genes work and interact to influence osteoporosis. Therefore, it is greatly significant to collect and analyze the studies involved in osteoporosis-related genes. Unfortunately, the information about all these osteoporosis-related genes is scattered in lots of extensive literature. Currently, there is no specialized database for easily accessing relevant information about osteoporosis-related genes and miRNAs.

Methods. We extracted data from literature abstracts in PubMed by text-mining and manual curation. Moreover, a local MySQL database containing all the data was developed with PHP on a Windows server.

Results. OsteoporosAtlas ( http://biokb.ncpsb.org/osteoporosis/), the first specialized database for easily accessing relevant information such as osteoporosis-related genes and miRNAs, was constructed and served for researchers. OsteoporosAtlas enables users to retrieve, browse and download osteoporosisrelated genes and miRNAs. Gene ontology and pathway analyses were integrated into OsteoporosAtlas. It currently includes 617 human encoding genes, 131 human non-coding miRNAs, and 128 functional roles. We think that OsteoporosAtlas will be an important bioinformatics resource to facilitate a better understanding of the pathogenesis of osteoporosis and developing better diagnosis, prevention and treatment strategies. 


\section{OsteoporosAtlas: a human osteoporosis-related gene 3 database}

4

5 Xun Wang ${ }^{1}$, Lihong Diao ${ }^{1}$, Dezhi Sun ${ }^{2}$, Dan Wang ${ }^{2}$, Jiarun $\mathrm{Zhu}^{2}$, Yangzhige $\mathrm{He}^{2}$, Yuan $\mathrm{Liu}^{2}$, $6 \mathrm{Hao} \mathrm{Xu}^{3}$, Yi Zhang ${ }^{3}$, Jinying Liu ${ }^{3}$, Yan Wang ${ }^{3}$, Fuchu He${ }^{4}$, Yang Li1 ${ }^{*}$, Dong Li ${ }^{1 *}$

7

$8{ }^{1}$ State Key Laboratory of Proteomics, Beijing Proteome Research Center, National Center for

9 Protein Sciences, Beijing Institute of Life omics, Beijing 102206, China

$10{ }^{2}$ School of Traditional Chinese Medicine, Beijing University of Chinese Medicine, Beijing, 11 China

Corresponding Author:

Yang $\mathrm{Li}^{1 *}$

Email address: liyang_bprc@163.com

Dong $\mathrm{Li}^{1 *}$

Email address: lidong.bprc@,foxmail.com

\section{Abstract}

Background. Osteoporosis is a common, complex disease of bone with a strong heritable component, characterized by low bone mineral density, microarchitectural deterioration of bone tissue and an increased risk of fracture. Due to limited drug selection for osteoporosis and increasing morbidity, mortality of osteoporotic fractures, osteoporosis has become a major health burden in aging societies. Current researches for identifying specific loci or genes involved in osteoporosis contribute to a greater understanding of the pathogenesis of osteoporosis and the development of better diagnosis, prevention and treatment strategies. However, little is known about how most causal genes work and interact to influence osteoporosis. Therefore, it is greatly significant to collect and analyze the studies involved in osteoporosis-related genes. Unfortunately, the information about all these osteoporosis-related genes is scattered in lots of 
30 extensive literature. Currently, there is no specialized database for easily accessing relevant 31 information about osteoporosis-related genes and miRNAs.

32 Methods. We extracted data from literature abstracts in PubMed by text-mining and manual 33 curation. Moreover, a local MySQL database containing all the data was developed with PHP on 34 a Windows server.

35

36

37

38

39

40

41

42

43

44

45

46

47

48

49

50

51

52

53

54

55

56

57

58

59

60

61

62

63

64

65

Results. OsteoporosAtlas (http://biokb.ncpsb.org/osteoporosis/), the first specialized database for easily accessing relevant information such as osteoporosis-related genes and miRNAs, was constructed and served for researchers. OsteoporosAtlas enables users to retrieve, browse and download osteoporosis-related genes and miRNAs. Gene ontology and pathway analyses were integrated into OsteoporosAtlas. It currently includes 617 human encoding genes, 131 human non-coding miRNAs, and 128 functional roles. We think that OsteoporosAtlas will be an important bioinformatics resource to facilitate a better understanding of the pathogenesis of osteoporosis and developing better diagnosis, prevention and treatment strategies.

\section{Introduction}

Osteoporosis is a common skeletal disease under strong genetic control (Ralston et al. 2006), whose characteristics are low bone mineral density (BMD), microarchitectural deterioration of bone tissue and increased risk of fracture (Kanis et al. 1994). Osteoporosis is generally induced by various causes, such as age, sex, genetic factors, a range of hormones and environmental factors. Moreover, genetic factors not only have strong control over osteoporosis and its associated phenotypes, but may also influence sensitivity to hormonal and environmental factors (Eisman 1999). Osteoporosis can be divided into primary, secondary, and idiopathic types.

Primary osteoporosis is more common and mostly caused by old age and postmenopause (Ji et al. 2015). Secondary osteoporosis is most commonly caused or exacerbated by medication exposures or other disorders (Stein et al. 2003). Idiopathic osteoporosis is a type of rare osteoporosis that often occurs in young, healthy individuals who are not postmenopausal or have other, identifiable secondary causes of osteoporosis (Heshmati \& Khosla 1998). The clinical outcomes of osteoporosis are fracture of hip, wrist, spine and other types. Hip fracture, an international barometer of osteoporosis, is the most severe osteoporotic fracture, $10-20 \%$ more women with hip fracture die than expected for age within the first year, and the mortality rate in men with hip fracture is even higher (Cummings \& Melton 2002). Menopausal estrogen deficiency is one of the most important reasons why women are more susceptible to osteoporosis. In addition, men generally have greater cortical mass and larger bone size than women (Richelson et al. 1984). Alarmingly, osteoporosis affects over 200 million individuals worldwide and the incidence of osteoporotic fractures is expected to rise by $50 \%$ over the next decade (Burge et al. 2007). With the aging of the population in recent years, osteoporosis is 
66 becoming an increasingly huge public health issue associated with increased mortality and

67 morbidity.

68 Osteoporosis is a polygenic disorder, generally determined by the combined effects of several

69 genes and environmental factors. Osteoporosis rarely occurs as the result of single gene

70 mutations. Twin and family studies have shown that genetic factors are extremely important in

71 the regulation of bone density, skeletal geometry, ultrasound properties of bone, bone turnover,

72 and risk of osteoporosis (Stewart \& Ralston 2000). Identification of specific loci or genes

73 determining osteoporosis will contribute to a better understanding of the pathogenesis of

74 osteoporosis and developing better diagnosis, prevention and treatment strategies. The current

75

76

77

78

79

80

81

82

83

84

85

86

87

88

89

90

91

92

93

94

95

96

97

98

99

100

101

102

103

104

efforts to identify osteoporosis loci or genes have mainly focused on three approaches: animal models, candidate gene approach and genome-wide scans (Huang et al. 2003). Genome-wide association studies have identified over 300 loci associated with BMD. However, little is known of how most causal genes interact with each other and the mechanisms to cause osteoporosis (AlBarghouthi \& Farber 2018). Recently, some studies have shown that how causal genes contribute to the pathogenesis of osteoporosis. For example, the research has shown that Siglec-15 genedeficient mice exhibit mild osteoporosis and Siglec-15 gene is involved in osteoclast differentiation induced by estrogen deficiency, which suggests Siglec-15 gene is a promising drug therapy target for postmenopausal osteoporosis and age-related osteoporosis (Kameda et al. 2015). C-Abl gene and Atm (ATM Serine/Threonine Kinase) gene result in osteoporosis by positively regulating osteoblast differentiation and bone formation, moreover mice lacking either of them shows osteoporosis, while P53 gene inhibits osteoporosis, which negatively regulates osteoblast differentiation and bone formation, and the knockout mouse shows osteosclerosis (Wang \& Li 2007). Sclerostin (SOST gene encoded) is an antagonist of WNT/ $\beta$-catenin signaling (canonical WNT pathway), which predominantly regulates osteoblast differentiation and plays important roles in regulating bone formation. Inactivating monoclonal antibodies against SOST, a inhibitor of the negative regulation of WNT/ $\beta$-catenin signaling, has been shown to be a candidate for the prevention and treatment of osteoporosis (Rossini et al. 2013).

MicroRNAs (miRNAs), a class of small ( 22bp) nucleotides, are single-stranded noncoding RNAs that regulate the expression of target genes by binding to their 3'-untranslated region (Carthew \& Sontheimer 2009). miRNAs play critical roles in the regulation of various biological processes by targeting mRNAs, including cellular differentiation and proliferation, apoptosis, and tissue development (Kim et al. 2009). An imbalance between osteoblastic bone formation and osteoclastic bone resorption plays a fundamental role in osteoporosis pathogenesis (Teitelbaum 2000). Recent studies discovered that miRNAs play an important role in the subtle equilibrium between bone formation and bone resorption by targeting various genes to regulate osteoblast and osteoclast differentiation and function (Ji et al. 2016). For example, Zhao et al have found that hsa-mir-21 overexpression aggravates osteoporosis by targeting RECK (Zhao et al. 2015). Hsa-mir-34a is a novel and pivotal suppressor of osteoclastogenesis and bone resorption, which blocks osteoporosis by inhibiting osteoclastogenesis and Tgif2 (Krzeszinski et

Peer) reviewing PDF | (2018:10:32180:1:1:NEW 28 Feb 2019) 
105

106

107

108

109

110

111

112

113

114

115

116

117

118

119

120

121

122

123

124

125

126

127

128

129

130

131

132

133

134

135

136

137

138

139

140

141

al. 2014). Hsa-mir-133a was shown to directly target Runx2 gene 3'-UTR when overexpressed in MC3T3, an osteoblast cell line. In addition, hsa-mir-133a was also shown to negatively regulate 3 potential osteoclast-related target genes, CXCL11, SLC39A1 and CXCR3. MiRNA expression analysis in human circulating monocytes shows that hsa-mir-133a is a promising biomarker for postmenopausal osteoporosis (Wang et al. 2012).

Currently, therapeutic drugs for osteoporosis include hormone replacement therapy, calcitonin, selective estrogen receptor, and bisphosphonates, etc. However, due to side-effects and high price, drug selection for osteoporosis is still limited (Martin \& Sims 2005). Therefore, research for identifying osteoporosis-related genes and their pathogenic mechanisms is greatly significant, which promote a better understanding of the pathogenesis of osteoporosis, identification of novel biomarkers, discovery of therapeutic targets and accurate treatment strategies. However, abundant information of previously confirmed osteoporosis-related genes is scattered in lots of extensive literature. Moreover, there is no specialized knowledge base dedicated to osteoporosisrelated genes collection, which makes it difficult to understand the pathogenesis of osteoporosis and develop new drug targets for osteoporosis. In order to address this obstacle, we established OsteoporosAtlas 1.0 (http://biokb.ncpsb.org/osteoporosis/) by literature-mining and manual curation, which currently contains 617 human osteoporosis-related genes, 131 human osteoporosis-regulated miRNAs, 84 biological process analysis, and 128 functional roles. Users can retrieve, browse and download osteoporosis-related genes and relevant information from OsteoporosAtlas. Moreover, gene ontology analysis, pathway analysis, and SNP term analysis were integrated into OsteoporosAtlas. In summary, we believe that OsteoporosAtlas will provide a comprehensive osteoporosis research resource for the pathogenesis of individual cases, discovery of drug target, novel diagnostic biomarker, individual treatment methods and precision medication.

\section{Materials \& Methods}

\section{Text Mining and Manual Curation}

We developed an ontology-based bio-entity recognizer (see the appendix for details). Abstract data of CRAFT 2.0 (Verspoor et al. 2012) corpus was used as the independent test set to evaluate the performance of our bio-entity recognizer by identifying gene/protein based on Protein Ontology (Natale et al. 2010). After evaluation, the precision (the number of correct entities identified divided by the number of entities identified), recall (the number of correct entities identified divided by the number of entities in the sample), F-measure (the harmonic mean of precision and recall) of our bio-entity recognizer for identifying gene/protein were respectively $0.959,0.802$ and 0.874 , indicating it is of great performance for entity recognition (both the evaluation program and result was available at http://biokb.ncpsb.org/osteoporosis/Public/file/craft_test.rar ). Therefore, we performed a 
142 comprehensive search for osteoporosis-related literature abstracts in PubMed to extract

143 osteoporosis-related genes by using the bio-entity recognizer. Specifically, it is divided into three

144 steps (Figure 1A):

145 First, data collection: we collected 79,947 sentences in 7,358 abstracts containing the keywords

146 of "osteoporosis", "osteoporoses", "bone loss, age-related", "age-related bone loss", and

147 "perimenopausal bone loss".

148 Second, candidate data extraction: 1,628 osteoporosis-related candidate genes/microRNAs and

149 13,264 candidate evidence sentences (Supplementary material: TableS1.xlsx) were extracted by

150 using our bio-entity recognizer.

151 Third, manual curation: our experts manually curated all candidate genes and evidence sentences,

152527 encoding genes and 131 miRNAs were finally confirmed as human osteoporosis-related

153 genes. In addition, we integrated osteoporosis-related genes confirmed in DisGeNET (Piñero et

154 al. 2015) (Figure 1B), an integrated comprehensive platform with information about human

155 disease-associated genes and variants. In summary, our database consists of 617 osteoporosis-

156 related encoding genes, 131 osteoporosis-regulated miRNAs (non-coding RNA).

157 Gene Annotation

158 In order to facilitate the deep interpretation of the association between osteoporosis and related 159 genes, we annotated each gene by the basic gene annotation files ("gene2refseq") from NCBI 160 FTP site with the information of gene synonyms symbol, genetic location, gene full name, 161 chromosome, gene type, reference sequence information and chromosomal location. We 162 obtained gene ontology annotation for each gene from the Gene Ontology Annotation database 163 (GOA) (Camon et al. 2004) and gene-pathway mapping relationship from the Reactome database 164 (Fabregat et al. 2015). The database of short genetic variation (dbSNP) (Sherry et al. 2001) was 165 used to map SNPs to gene by the publications' PMIDs (PubMed sole Identifier), which makes it 166 easier to access to genome-wide association study and helps researchers to improve the 167 understanding of the genetic background of osteoporosis. Mapping and annotating are done by 168 using public databases of Entrez gene (Maglott et al. 2005),Ensembl (Flicek et al. 2010), UniProt 169 (Bairoch et al. 2005), Antibodypedia (Björling et al. 2008) and neXtProt (Lane et al. 2011).

170

171 Results

\section{Database Service and implement}

173 All osteoporosis-related genes and their relevant information were deposited into a local MySQL 174 database. The website of OsteoporosAtlas (http://biokb.ncpsb.org/osteoporosis/) was developed 
175 with PHP on a Windows server. All data about OsteoporosAtlas is accessible for all users

176 without registration or login.

\section{Database Navigation and Search}

178 In order to provide a user-friendly web interface for searching and browsing, we designed five 179 sections of functions for our database (Figure 2A) and provide three query approaches.

180 For the search by gene name, users can input a gene symbol in the "Gene Name" search box 181 (Figure 2B). After clicking the "search" button, user can get detailed gene annotation for osteoporosis (Figure 2D), including functional role (whether this gene is a biomarker/drug target), supporting literature evidence, SNP information from the database of short genetic variation (dbSNP), gene ontology (GO) terms from Gene Ontology Annotation database (GOA), the protein description from database UniProtKB, pathway terms from Reactome analysis, gene expression information from Expression Atlas, protein expression information from Human Protein Atlas (HPA), and the regulated miRNAs information from miRBase database (Kozomara \& Griffiths-Jones 2013). Clicking the number of abstracts as evidence will lead to the detailed info of the evidence abstracts (Figure 2F, 2G), and clicking the number of the involved biological process will lead to the view of the involved biological processes (Figure 2E).

The section of 'Browse \& Download' provides four different approaches, including genes, miRNAs, biological processes and functional roles. The current version of database contains 617 genes, 131 miRNAs, 84 biological processes and 128 functional roles. All the information can be downloaded. In the section of "Feedback", we provide a submitting function. If users found osteoporosis-related genes which are absent from our database, they can submit this gene to our database.

\section{7}

198

199

200

201

202

203

204

205

206

207

208

209

210

211

\section{Database Use Case}

Users can find SOST gene by searching gene name, protein sequence or nucleotide sequence in our database. Four functional sections are provided in the search results interface (Figure 2B): Relevant information about gene interface (Figure 2D) provides functional role (whether this gene is a biomarker/drug target), supporting literature evidence, relevant information about genome-wide association study from the database of short genetic variation (dbSNP), gene ontology (GO) terms from Gene Ontology Annotation database, the protein description from UniProtKB database, pathway terms from Reactome analysis, gene expression information from Expression Atlas, protein expression information from Human Protein Atlas (HPA). Evidence interface (Figure 2F, 2G) provides the literature which supports the association of SOST gene with osteoporosis. Biological process interface (Figure 2E) provides gene ontology terms associated with SOST gene. Searching the name of the biological process can browse the genes in this biological process.

Users can find hsa-mir-100 by searching miRNA name in our database. Three functional sections are provided in the search results interface (Figure 2C). Clicking hsa-mir-100 will jump to 
212 miRBase database, an online resource for miRNA sequence and annotation (Figure 2H).

213 Evidence interface provides the literature which supports the association of hsa-mir-100 with

214 osteoporosis (Figure 2I, 2J).

215

\section{Discussion}

217 The systematic collection of OsteoporosAtlas 1.0 database provides an overview of human 218 osteoporosis-related genes. Reactome analysis shows that these genes are involved in pathways

219 of signal transduction, immune system, gene expression (transcription), extracellular matrix 220 organization, etc (Figure 3A). Gene ontology analysis using Panther indicates that the most 221 common class of osteoporosis-related genes belongs to signaling molecule, followed by 222 hydrolase/nucleic acid binding, receptor/transcription factors, enzyme modulator, oxidoreductase, 223 transferase, transporter, etc (Figure 3B). The Reactome and gene ontology analysis could help 224 biologists to better understand the functional relevance of these genes and guide the experiment. 225 In addition, the existence of many potential pharmacological targets in these pathways we 226 analyzed makes it attractive for osteoporosis drugs discovery. All the results show the value of 227 our collection, demonstrating that OsteoporosAtlas 1.0 database will greatly benefit exploration 228 of pathogenesis and treatment of osteoporosis.

229 OsteoporosAtlas 1.0 also has community curation feature. All users can log in to provide their 230 feedback, confirm or reject the evidence sentence by clicking the "Yes" or "No" button. We will 231 update the database periodically according to users' feedbacks.

232 We identified 94 drug target genes with definite literature evidences in our database. The 233 existing osteoporosis treatment drugs mainly delay bone loss by inhibiting bone resorption or 234 promoting bone formation. However, due to the high price and side effects, the osteoporosis 235 drugs promoting bone formation without side-effects are still in the basic clinical research stages. 236 So our database will help discover new drugs for the treatment of osteoporosis.

237

238

\section{Conclusions}

239 In conclusion, OsteoporosAtlas 1.0 collects 617 osteoporosis-related encoding genes and 131 240 miRNAs, which is the first database specially to present a comprehensive list of osteoporosis241 related genes obtained from published literature. We believe that OsteoporosAtlas 1.0 will be 242 widely used as it can provide facilities for scientists and clinicians in searching the literature on 243 osteoporosis-related genes and their function in diseases. 
246 The detailed description of methods:

247 (1). Construction and processing of vocabulary

248 It is necessary to construct different types of vocabularies for natural language processing and 249 semantic recognition, including stop words, high frequency words, domain words and 250 abbreviations. Stop words are mainly from PubMed, MetaMap (Aronson 2001), and Python 251 NLTK (Loper \& Bird 2002). High frequency words are mainly from PIR (Protein Information 252 Resource (Barker et al. 2000)) Common English, General Names, Common English of PIR 253 iProLINK (Hu et al. 2004) Entity Recognition, and Google 1000 English. Domain words are 254 mainly from the gene/protein, compound, enzyme, and other biochemical naming dictionary in 255 PIR. Abbreviations are mainly from the open source software BADREX (Gooch 2012) in Github. 256

257 (2). Collection and processing of literature

258 The PubMed abstracts were downloaded in XML format by NCBI E-Utilities API (Sayers 2010) 259 and parsed by python script. The database currently contains approximately 26 million abstracts.

260 (3). Collection and processing of ontology

261 A high-quality dictionary of human genes/proteins and their synonyms is mainly based on 262 Protein Ontology (Natale et al. 2010) and bioThesaurus (Duek et al. 2011). And the human 263 protein ID of UniProtKB is used as a standardized ID.

264 (4). Entity recognition

265 Indexer (python script) is used to build indexes for the PubMed abstracts. Analyzer (python 266 script) is used to perform synonym expansion on the ontology. Indexes and synonyms were 267 combined to perform span near match and entity recognition.

268

269

\section{Supplementary material}

270 Supplementary data are available at PeerJ Online.

271

\section{Acknowledgements}

273 We would like to thank the anonymous reviewers for their useful comments and suggestions on 274 this manuscript.

275 


\section{References}

277

278

279

280

281

282

283

284

285

286

287

288

289

290

291

292

293

294

295

296

297

298

299

300

301

302

303

304

305

306

307 308

Al-Barghouthi BM, and Farber CRJTiG. 2018. Dissecting the Genetics of Osteoporosis using Systems Approaches.

Aronson AR. 2001. Effective mapping of biomedical text to the UMLS Metathesaurus: the MetaMap program. Proceedings of the AMIA Symposium: American Medical Informatics Association. $\mathrm{p} 17$.

Bairoch A, Apweiler R, Wu CH, Barker WC, Boeckmann B, Ferro S, Gasteiger E, Huang H, Lopez R, and Magrane MJNar. 2005. The universal protein resource (UniProt). 33:D154-D159.

Barker WC, Garavelli JS, Huang H, McGarvey PB, Orcutt BC, Srinivasarao GY, Xiao C, Yeh LSL, Ledley RS, and Janda JFJNar. 2000. The protein information resource (PIR). 28:4144.

Björling E, Uhlén MJM, and Proteomics C. 2008. Antibodypedia, a portal for sharing antibody and antigen validation data. 7:2028-2037.

Burge R, Dawson-Hughes B, Solomon DH, Wong JB, King A, Tosteson AJJob, and research $\mathrm{m}$. 2007. Incidence and economic burden of osteoporosis-related fractures in the United States, 2005-2025. 22:465-475.

Camon E, Magrane M, Barrell D, Lee V, Dimmer E, Maslen J, Binns D, Harte N, Lopez R, and Apweiler RJNar. 2004. The gene ontology annotation (goa) database: sharing knowledge in uniprot with gene ontology. 32:D262-D266.

Carthew RW, and Sontheimer EJJC. 2009. Origins and mechanisms of miRNAs and siRNAs. 136:642-655.

Cummings SR, and Melton LJJTL. 2002. Epidemiology and outcomes of osteoporotic fractures. 359:1761-1767.

Duek PD, Gleizes A, Zwahlen C, Mottaz A, Bairoch A, and Lane LJB-O. 2011. CALOHA: A new human anatomical ontology as a support for complex queries and tissue expression display in neXtProt.

Eisman JAJEr. 1999. Genetics of osteoporosis. 20:788-804.

Fabregat A, Sidiropoulos K, Garapati P, Gillespie M, Hausmann K, Haw R, Jassal B, Jupe S, Korninger F, and McKay SJNar. 2015. The reactome pathway knowledgebase. 44:D481-D487.

Flicek P, Amode MR, Barrell D, Beal K, Brent S, Chen Y, Clapham P, Coates G, Fairley S, and Fitzgerald SJNar. 2010. Ensembl 2011. 39:D800-D806. 
309

310

311

312

313

314

315

316

317

318

319

320

321

322

323

324

325

326

327

328

329

330

331

332

333

334

335

336

337

338

339

340

341

Gooch PJapa. 2012. Badrex: In situ expansion and coreference of biomedical abbreviations using dynamic regular expressions.

Heshmati HM, and Khosla S. 1998. Idiopathic osteoporosis: a heterogeneous entity. Annales de medecine interne. $p$ 77-81.

Hu Z-Z, Mani I, Hermoso V, Liu H, Wu CHJCb, and chemistry. 2004. iProLINK: an integrated protein resource for literature mining. 28:409-416.

Huang Q-Y, Recker RR, and Deng H-WJOI. 2003. Searching for osteoporosis genes in the post-genome era: progress and challenges. 14:701-715.

Ji M-X, Yu QJCd, and medicine t. 2015. Primary osteoporosis in postmenopausal women. 1:9.

Ji X, Chen X, and Yu XJljoms. 2016. MicroRNAs in osteoclastogenesis and function: potential therapeutic targets for osteoporosis. 17:349.

Kameda Y, Takahata M, Mikuni S, Shimizu T, Hamano H, Angata T, Hatakeyama S, Kinjo M, and Iwasaki NJB. 2015. Siglec-15 is a potential therapeutic target for postmenopausal osteoporosis. 71:217-226.

Kanis JA, Melton III LJ, Christiansen C, Johnston CC, Khaltaev NJJob, and research m. 1994. The diagnosis of osteoporosis. 9:1137-1141.

Kim VN, Han J, and Siomi MCJNrMcb. 2009. Biogenesis of small RNAs in animals. 10:126.

Kozomara A, and Griffiths-Jones SJNar. 2013. miRBase: annotating high confidence microRNAs using deep sequencing data. 42:D68-D73.

Krzeszinski JY, Wei W, Huynh H, Jin Z, Wang X, Chang T-C, Xie X-J, He L, Mangala LS, and Lopez-Berestein GJN. 2014. miR-34a blocks osteoporosis and bone metastasis by inhibiting osteoclastogenesis and Tgif2. 512:431.

Lane L, Argoud-Puy G, Britan A, Cusin I, Duek PD, Evalet O, Gateau A, Gaudet P, Gleizes A, and Masselot AJNar. 2011. neXtProt: a knowledge platform for human proteins. 40:D76-D83.

Loper E, and Bird SJapc. 2002. NLTK: the natural language toolkit.

Maglott D, Ostell J, Pruitt KD, and Tatusova TJNar. 2005. Entrez Gene: gene-centered information at NCBI. 33:D54-D58.

Martin TJ, and Sims NAJTimm. 2005. Osteoclast-derived activity in the coupling of bone formation to resorption. 11:76-81.

Natale DA, Arighi CN, Barker WC, Blake JA, Bult CJ, Caudy M, Drabkin HJ, D'eustachio P, Evsikov AV, and Huang HJNar. 2010. The Protein Ontology: a structured representation of protein forms and complexes. 39:D539-D545. 
342 Piñero J, Queralt-Rosinach N, Bravo A, Deu-Pons J, Bauer-Mehren A, Baron M, Sanz F, and

343

344

345

346

347

348

349

350

351

352

353

354

355

356

357

358

359

360

361

362

363

364

365

366

367

368
Furlong LIJD. 2015. DisGeNET: a discovery platform for the dynamical exploration of human diseases and their genes. 2015.

Ralston SH, de Crombrugghe BJG, and development. 2006. Genetic regulation of bone mass and susceptibility to osteoporosis. 20:2492-2506.

Richelson LS, Wahner HW, Melton III L, and Riggs BLJNEJoM. 1984. Relative contributions of aging and estrogen deficiency to postmenopausal bone loss. 311:1273-1275.

Rossini M, Gatti D, and Adami SJCti. 2013. Involvement of WNT/ $\beta$-catenin signaling in the treatment of osteoporosis. 93:121-132.

Sayers EJEPUHBNCfBI. 2010. A General Introduction to the E-utilities.

Sherry ST, Ward M-H, Kholodov M, Baker J, Phan L, Smigielski EM, and Sirotkin KJNar. 2001. dbSNP: the NCBI database of genetic variation. 29:308-311.

Stein E, Shane EJE, and America mcoN. 2003. Secondary osteoporosis. 32:115-134, vii.

Stewart T, and Ralston SJJoE. 2000. Role of genetic factors in the pathogenesis of osteoporosis. 166:235-246.

Teitelbaum SLJS. 2000. Bone resorption by osteoclasts. 289:1504-1508.

Verspoor K, Cohen KB, Lanfranchi A, Warner C, Johnson HL, Roeder C, Choi JD, Funk C, Malenkiy $\mathrm{Y}$, and Eckert MJBb. 2012. A corpus of full-text journal articles is a robust evaluation tool for revealing differences in performance of biomedical natural language processing tools. 13:207.

Wang X, and Li BJljobsl. 2007. Genetic studies of bone diseases: evidence for involvement of DNA damage response proteins in bone remodeling. 3:217.

Wang Y, Li L, Moore BT, Peng X-H, Fang X, Lappe JM, Recker RR, and Xiao PJPo. 2012. MiR133a in human circulating monocytes: a potential biomarker associated with postmenopausal osteoporosis. 7:e34641.

Zhao W, Dong Y, Wu C, Ma Y, Jin Y, Ji YJM, and biochemistry c. 2015. MiR-21 overexpression improves osteoporosis by targeting RECK. 405:125-133. 


\section{Figure 1}

Figure1 The construction workflow of OsteoporosAtlas 1.0 database.

(A) The workflow of OsteoporosAtlas 1.0 database construction; (B) The comparison of osteoporosis-related genes between OsteoporosAtlas 1.0 database and DisGeNET database.

A

The workflow of OsteoporosAtlas

\section{Data collection}

keywords of 'osteoporosis' 'age-related bone loss' and their lexical variants 7358 osteoporosis-related abstracts 79,947 sentences

\section{Data extraction}

extraction by our bio-entity recognizer

13,264 sentences in 7,358 PubMed abstract 1,494 encoding genes, 134 microRNAs

\section{Manual curation}

Three rounds of manual curation

617 osteoporosis-related genes

131 microRNAs
B

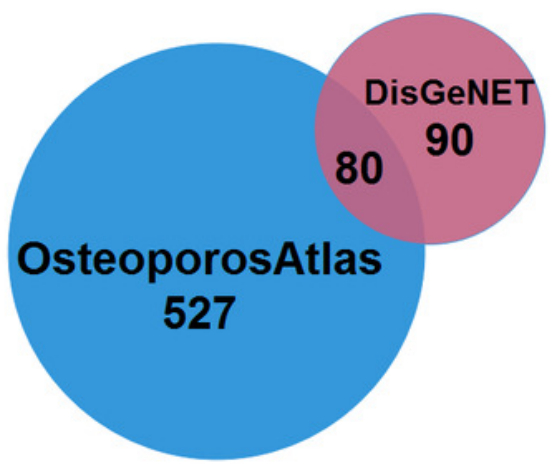




\section{Figure 2 (on next page)}

Figure2 The outline for searching in OsteoporosAtlas 1.0 website.

(A) Five functional sections of the database. (B) Users can submit a gene name to the "Gene Name" search box and the search result will be returned, including the information of Gene, related biological processes, functional role, and evidence. (D) After clicking the gene name, users can get more specific information about this gene on the detailed page. (E) After clicking on the number of biological processes, users can scan the biological processes involved by this gene. ( $F, G)$ After clicking the number of evidence, the original abstract will be displayed with highlighted matched sentence and matched keywords.(C) Users can submit a microRNA name to the "MicroRNA Name" search box and the search result will be returned, including the information of microRNA, functional role and support evidence. $(H)$ After clicking the microRNA name, users can get more specific information about this microRNA in the detailed page. $(I, G)$ After clicking the number of the evidence, the original abstract will be displayed with highlighted matched sentence and matched keywords. 


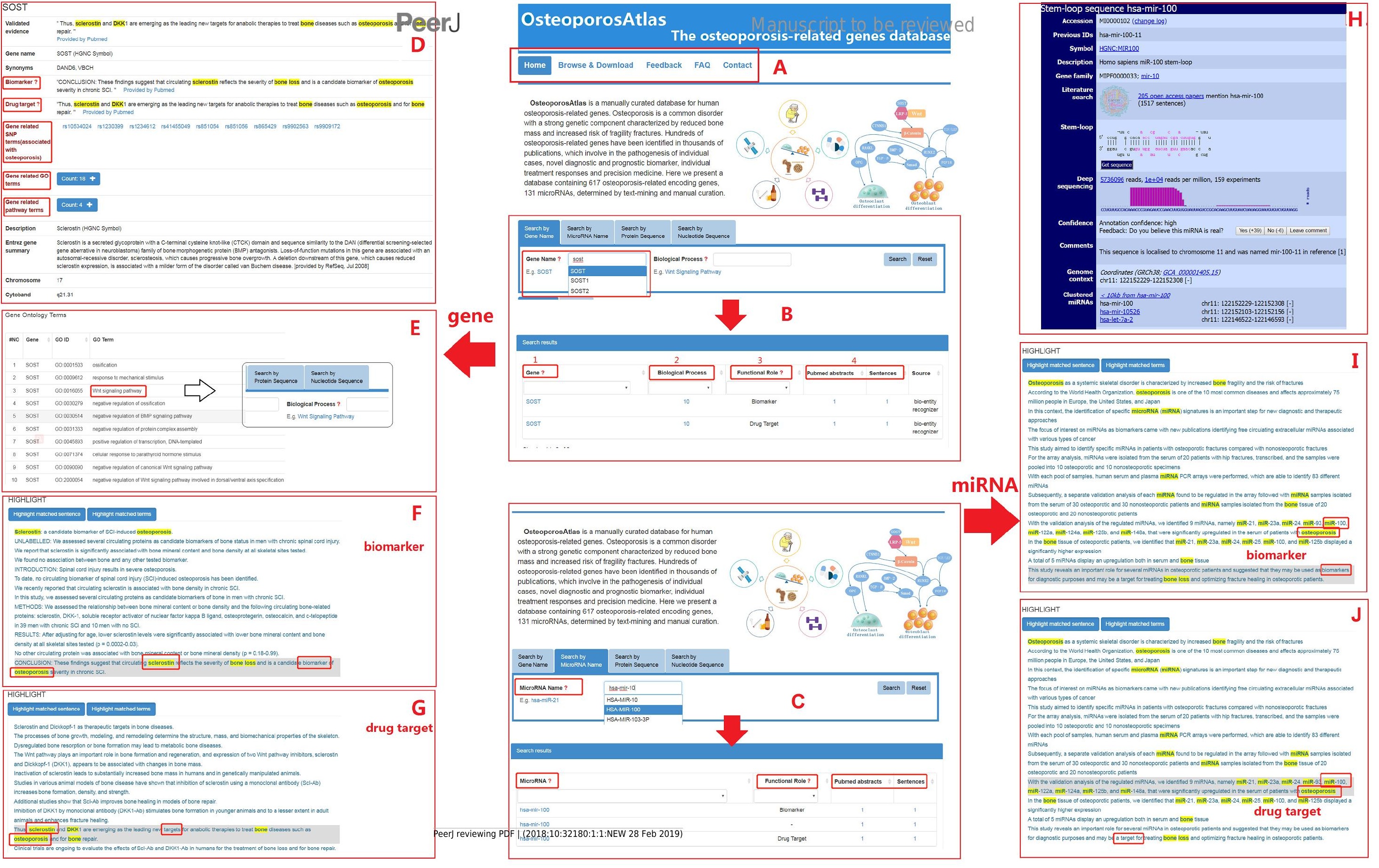


Figure 3

Figure 3 Bioinformatics analysis of genes associated with osteoporosis.

(A) Biological pathway analysis using Reactome (http://www.reactome.org/). (B) Protein class analysis using PANTHER ( http://www.pantherdb.org).

A

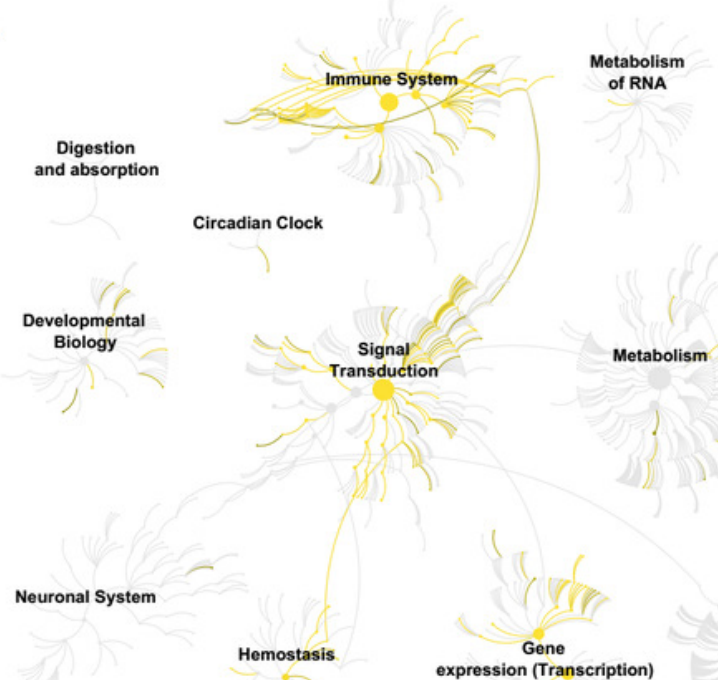

Chromatin

organization

$\begin{array}{lll}\text { DNA Replication } & \text { Cell Cycle } & \begin{array}{c}\text { Programmed } \\ \text { Cell Death }\end{array}\end{array}$

DNA Repair

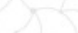

\section{DNARopair}
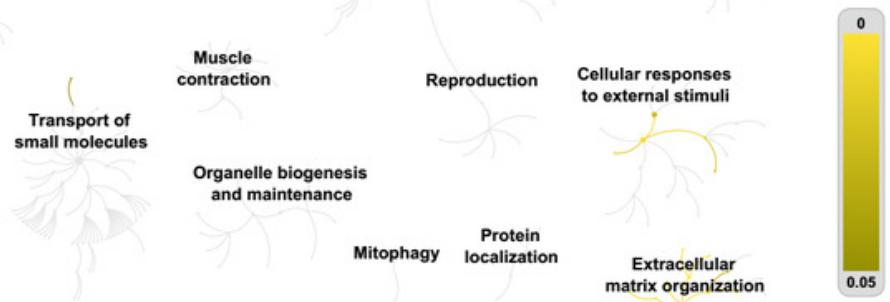

Hemostasis

expression (Transcription)

Metabolism

of proteins

Vesicle-mediated

transport

sease

communication

B Protein classification of 617 osteoporosis-related genes
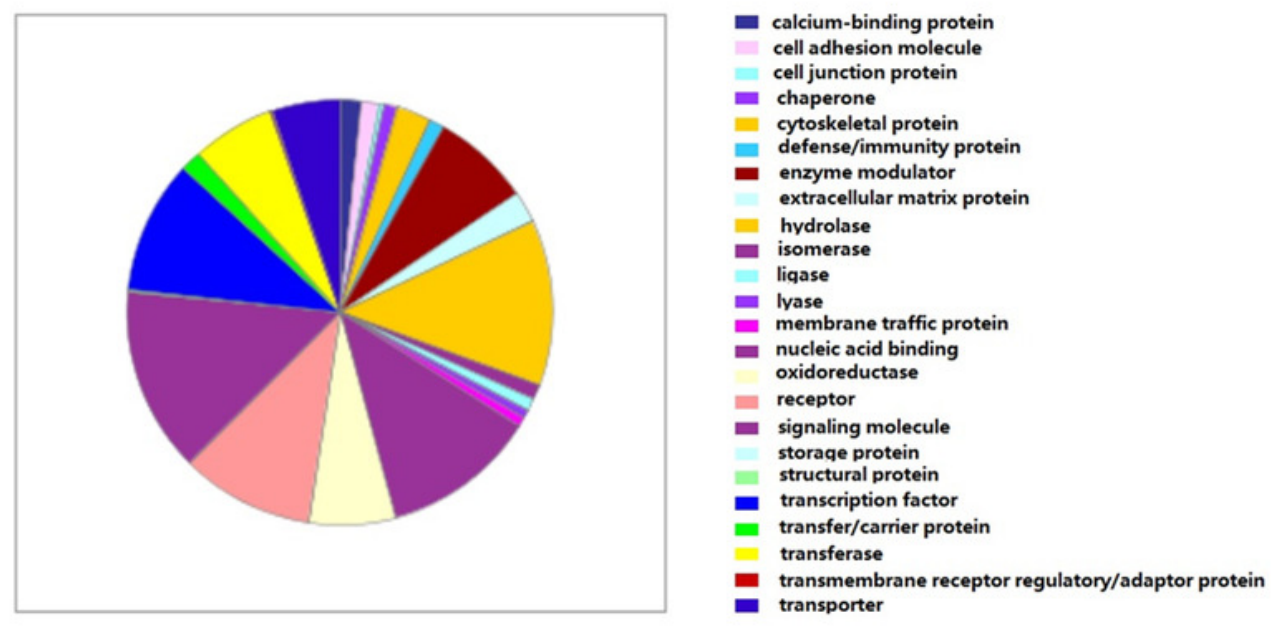